\title{
THE EFFECTIVENESS OF ACTIVE MODIFIED PRESURGICAL NASOALVEOLAR APPLIANCE IN ALIGNMENT OF PREMAXILLA OF COMPLETE BILATERAL CLEFT LIP AND PALATE INFANTS
}

\author{
Rana A. Negm ${ }^{1} M s C$, Mahmoud KH. Abdel Razek ${ }^{2} P h D$, Faten E. Mohamed. ${ }^{2} P h D$, \\ Ahmed S. Habib ${ }^{3} P h D$
}

\begin{abstract}
INTRODUCTION: Complete bilateral cleft lip and palate CBCLP is considered the most difficult form of cleft cases nowadays. Nasoalveolar molding enhances the esthetic and functional results of cleft infants. NAM works on the principle of sculpting and molding of the alveolus and adjacent soft tissues.

OBJECTIVES: The aim of this study was to assess the efficiency of a presurgical modified screw activated NAM appliance in retruding the premaxilla for CBCLP infants.

MATERIALS AND METHODS: Fifteen CBCLP infant were included in the study. Preliminary maxillary impressions were made and the produced casts were scanned to obtain three-dimensional virtual image which was used to plan the design of the appliance. Two-piece acrylic active appliance with reverse expansion screw was used to retrude the premaxilla. The appliance was retained in place using niklel chromium side extension arms and head cap. When the cleft gap was closed to be suitable for surgical repair, primary lip repair was performed. Impressions were made again; the casts were scanned to be converted into 3D models on which linear analysis of the cleft gap was done.

RESULTS: The results showed statistically significant decrease in the cleft gap following active alveolar molding.

CONCLUSION: Active NAM appliance can effectively retrude and correct the severely rotated and deviated premaxilla providing better esthetic results.
\end{abstract}

KEYWORDS: naso-alveolar molding (NAM), BCLP, active appliance, side-extension arms.

1. Assistant Lecturer of Prosthodontics, Faculty of dentistry, Alexandria University

2. Professor of Prosthodontics, Faculty of Dentistry, Alexandria University

3. Assistant Professor of Maxillofacial and Plastic Surgery, Faculty of dentistry, Alexandria University

*Corresponding author

Ramnegm87@yahoo.com

\section{INTRODUCTION}

Cleft lip and palate (CLP) is one of the most common congenital facial anomalies affecting one in every 700-1000 newborns worldwide (1). The anomaly is characterized by the lack continuity of tissues forming the lip, alveolus, and soft and hard palate (2).

Normal development of the palate depends on proper migration, growth, differentiation, and apoptosis of the cells, and occurs in three major stages: vertical growth of the shelves down toward the sides of the tongue, elevation of the palatal shelves to acquire a horizontal position as the mandible lengthens, and fusion of the palatal shelves to form the transient midline epithelial seam, which ultimately undergoes epithelial to mesenchymal transition (3).

Cleft palate can arise due to an error in any of these stages. These defects can be grouped into five categories: failure of palatal shelf formation, fusion of the palatal shelf with the tongue or mandible, failure of palatal shelf elevation, failure of palatal shelves to meet post-elevation, and persistence of the medial edge epithelium (4).

The etiology of cleft lip and/or cleft palate, although still largely unknown, has been extensively studied in industrialized countries and is suggested to be heterogeneous with increasing evidence that both genetic and environmental factors are operating. According to Amaratunga et al (5), the majority of clefts of the lip and palate are believed to have a multifactorial etiology with several genetic and environmental factors interacting to interrupt the normal fusion of the palate In a meta-analysis reviewed in 2013 (6), it was found that folic acid and smoking have high influence of oro-facial clefts outcomes. Also the exposure to some environmental factors such as sunshine, ultraviolet radiation, and ambient nitrogen 
oxides (nitrogen oxide, nitrogen monoxide, and nitrogen dioxide), sulfur dioxide, and ozone were found to be highly correlated to having cleft lip and palates infants (7).

Although the management of cleft lip and palate infants presents many challenges, a team including different specialists can maximize the benefit of the treatment and provide the optimum esthetical and functional outcome.

For Management of cleft lip and palate, several options can be included such as: lip adhesion surgery (8), Lip tapping without surgical intervention (9), Presurgical infant oral appliances (PSIO) and presurgical Nasoalveolar molding (NAM) appliance which was considered the changing point in the treatment of cleft infants enhancing the esthetic of both lips and nose (10). NAM appliances can either be active, semiactive or passive.

Active maxillary appliances move alveolar cleft segments in a predetermined manner with controlled forces; whereas passive appliances deliver no force, but act as a fulcrum upon which the forces mold the alveolar segments in a non-predictable fashion, maintain the distance between the two maxillary segments, while external force is applied to the primarily to reposition it posteriorly. Different mechanical means of retention can be used with NAM appliances such as elastic chains, screws, springs and plates (11).

In 2010, Mishra et al (12) conducted a study to evaluate the effect of naso-alveolar molding and lip reshaping on 15 infants. The infants were less than 1 year old. The study included infants with both unilateral and bilateral cleft lip and palate. They used three pieces acrylic plate with reverse expansion screw, although their major interest was the nasal remodeling, they found that the maxillary segments had been realigned to a favorable position. Also in 2013, Retnakumari et al (13) evaluated the change in the maxillary dimensions using an active jack- screw activated appliance. They find that the maxillary segments had been better aligned after surgical closure

The most widely used method of NAM retention is the extraoral tape. In 2016, Demet et al (14) conducted a study to analyze the adaptation, challenges, and satisfaction with NAM reported by caretakers of babies with cleft lippalate (CLP). They found that during NAM treatment, the most encountered problem was the cheek skin irritation due to tapes (39 babies, 73.6\%).

On the other hand, Grayson and Santiago (15) stated that "In 1686, Hoffman described the use of a head cap with arms extended to the face to retract the pre-maxilla and narrow the cleft. Also in 1561, Franco described a head cap for extra oral therapy”. In 1980 Abdel Razek (16) used 2mm wire to form side extension arms adapted over the checks of the infant and retain the appliance in place. This technique prevented the tissue irritation arises from the tape.

The aim of this study was to evaluate the efficiency of modified two piece active appliance in the retraction of premaxilla of bilateral complete cleft lip and palate infants
The Null hypothesis was that using modified presurgical active appliance has negative effect in re-aligning the premaxilla for CBCLP infants.

\section{MATERIALS AND METHODS}

\section{Study settings}

A sample of 15 male infants ranged from 1-3 weeks old who attended to the maxillofacial surgery department, Faculty of Dentistry, Alexandria University were randomly selected based on certain eligibility criteria. According to Hertzog (17)2008, 15 infants represented the minimal sample size aimed to investigate how bilateral cleft lip and palate cases responded differently to presurgical orthopedics and primary lip repair based on premaxillary characteristics.

Patient selection criteria

Infants included into this study the following criteria

Inclusion criteria

Non- syndromic complete bilateral cleft lip and palate infants less than 1 month of age.

Protruded, deviated or rotated premaxilla.

\section{Exclusion criteria}

Simple, operable cases.

Cleft infants with semilunar nasal band (incomplete clefts).

Syndrommic cases.

Patient approval and preparation

The selected infants were referred by the maxillofacial surgeon to the removable prosthodontics department, Faculty of Dentistry, Alexandria University for completing the examination, proposing a treatment plan and explaining the concept of the treatment to the parents of each child.

Informed consent: a written informed consent was obtained from all infants' parents who accepted to share in this study after explaining the procedure to them. It was also mentioned that the patient has the right of withdrawal fromm the study without any consequences. Ethical approval for this study was obtained from Research Ethics Committee, Faculty of Dentistry, Alexandria University, Egypt.

Fabrication of the prosthetic appliance

A preliminary impression was made using stock tray with heavy body poly-vinylsiloxane impression.

After complete setting of the impression material, the impression was retrieved and the mouth was examined for residual material.

The impression was poured using hard stone to get a preliminary cast and to construct a self-cure custom made tray.

Medium viscosity rubber base impression material was used for making final impression which was poured to obtain a master cast.

The infant was held in an inverted position to keep the tongue forward, keep the airway patent and to prevent suffocation from any excess impression material (Figure1). The master cast was duplicated. The original master cast was used to construct the modified active naso-alveolar molding 
device while the duplicate cast was kept as a permanent record to compare the dimensional changes after treatment. Designing phase

The master cast was scanned to obtain 3D virtual model which was used for measuring the angle of deviation and the amount of separation of premaxilla in relation to the vomer. The maxillofacial surgeon suggested the future position of the premaxilla according to which the amount of screw opening was adjusted.

Two lines were drawn; one vertically in the center of vomer and the other in the center of the premaxilla. The point of intersection between the two lines was considered the angle of deviation. This angle was calculated and provided to the laboratory technician to adjust the degree of screw inclination (Figure 2).

Prosthetic phase

The two piece appliance was fabricated in clear acrylic resin which was a modification of the active screw orthopedic appliance used by Oosterkamp et al (2005) (18). It was modified by adding the side tension arms as described in 1980 by Abdel Razek (16) (extra oral retentive wings).

The fabrication of the presurgical appliance

The master cast was prepared by blocking out the vault of the entire length of the cleft and the vomer was relived using base plate wax then sodium alginate (Pyrax cold mold seal, indiamart. India) was applied as a separating medium.

A modification of the active appliance was done by adding extra-oral retentive wings (side tension arms). Readymade facebow with two straight side length of hard, rounded, of $2 \mathrm{~mm}$ diameter, $15 \mathrm{~cm}$ length Nickel-Chrome wire with omega loops (at the end of the wire) was used to form extraoral wings to be used as a retentive aid to maintain the prosthesis in situ. For all infants, Size one face-bow was suitable for the size and contour of the archs of the infants in this age. The wire was bent to form the extra-oral wings and adapted on the cast to follow the contour of the labial and buccal surface of the alveolar ridge.

A reverse expansion screw was added to the cast following the proposed plan (Figure 3) as following

It was opened according to the needed distance to retract the premaxilla.

A small piece of a self-cure clear acrylic resin was added to both ends of the screw then attached to the palatal side of the premaxilla according to the previously determined angle.

Base plate wax was adapted on the prepared master cast in the form of two pieces and trimmed to the proper extent of the functional sulcus (Figure 4).

The cast was flasked using custom made gypsum mold as a flask. This gypsum mold was fabricated for each cast to hold the extra-oral wire wings in place and to prevent any movement during packing the cold-cure clear acrylic resin material

The appliance was packed, finished and polished according to the conventional method then it was ready to be inserted into the infant's mouth.
The oral cavity was carefully examined for any blanching of soft tissues with proper relief using pressure indicating paste. Retention of the appliance

Vaseline was placed on the infants face under the omega loops. A small piece of cold cure clear acrylic resin was added and adapted to the loops and manipulated to follow the checks contour. Care was taken to avoid traumatizing the infants'skin.

After the complete polymerization of the acrylic parts, they were finished and polished (Figure 5).

A cloth head-cap was used as a retentive aid. It was adjusted to suit the size of the infant's head and attached to side tension arms

Appliance insertion

The appliance was activated and inserted to the infant's mouth and instructions related to the insertion and removal of the appliance were given to the mother.

Recall visits once a week was scheduled to activate the screw (anti-clockwise) until the cleft gap was ready for surgery. According to the manufacturer's instructions, eachturn closes the expansion screw by quarter millimeter. On the recall visits, after any adjustments, the oral cavity was examined for any inflammation.

Follow-up

In each recall visit, medium body rubber base impression was made and poured. The obtained models were scanned and the dimension of the premaxilla and the gap of the cleft were measured and compared.

Surgical procedures

The primary surgical closure of the lip was performed when the premaxilla resume a correct close position to the other maxillary segment. All the surgeries followed the same technique of closure. The surgery was performed under general anesthesia with endotracheal entubation, supine position with the neck extended. The child, the face was disinfected with Betadine with the eyes being protected by adhesive tape. Straight line closure technique was followed in the surgery. No tension was exerted on the tissues or on the sutures.

Straight line closure techniques was applied to all cases in which bilateral rotation advancement flaps were raised and sutured to the prolabium flapin three layers closure; oral, muscle and the mucosa. The anterior palatal third (premaxilla) was repaired bilaterally and simulatenoeusly with the bilateral lip repair using vomerine flaps at the midline sutured to nasal flap laterally.

Assessment phase

Pre-surgical analysis of the maxillary casts to assess the change in position of premaxilla and geometry of maxillary segments was done using SOLIDWORKS 10.0 software. The $3 \mathrm{D}$ quantitative maxillary geometric analysis was performed at three different time intervals as following:

M0: before appliance fabrication.

M1: 6-8 weeks of treatment

M2: 12- 16 weeks of treatment (at time of the surgery). 
Similar to Agrawal and Patel (19) in 2019, reference points and lines which were based on the anatomic structured were identified on all casts. The following land marks were added and the corresponding distances were measured (Figure 6).

$\mathrm{AB}$ The width of the premaxilla (measured between the widest two points).

EF The distance between the premaxilla and the posterior part of the vomer.

AC and BD right and left cleft gaps (The distance between the premaxilla and the alveolar ridges).

Statistical Analysis

Data were collected and entered to the computer using SPSS (Statistical Package for Social Science) program for statistical analysis (20). Kolmogorov-Smirnov test of normality revealed no significance in the distribution of the variables, so the parametric statistics was adopted (21). Data were described using minimum, maximum, mean, standard deviation and 95\% CI of the mean (22). Comparisons were carried out between two studied independent normally distributed variables using independent sample t test (23). When Levene's test for equality of variances is significant, Welch's t-test is used, which is an adaptation of Student's ttest (24), and is more reliable when the two samples have unequal variances and unequal sample sizes (25).

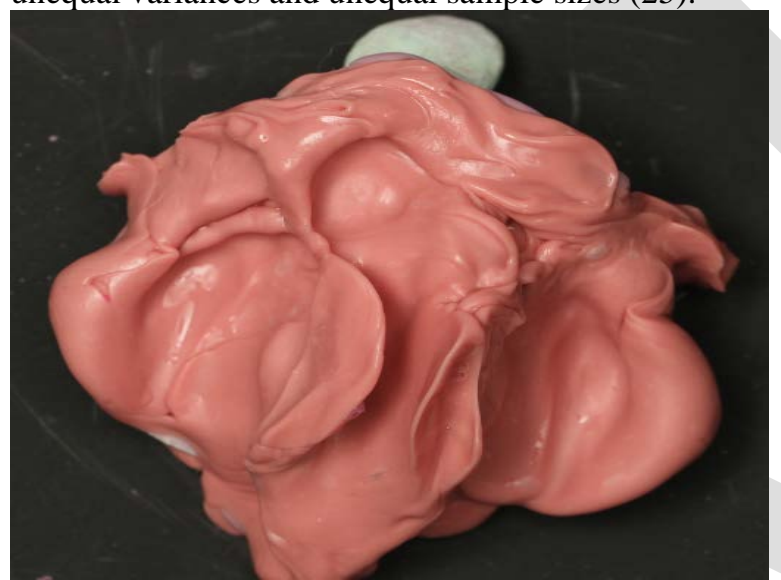

Figure (1): Final poly-vinylsiloxane impression.

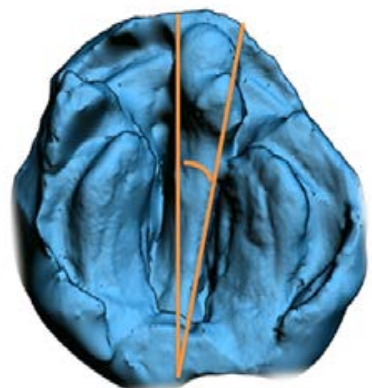

A

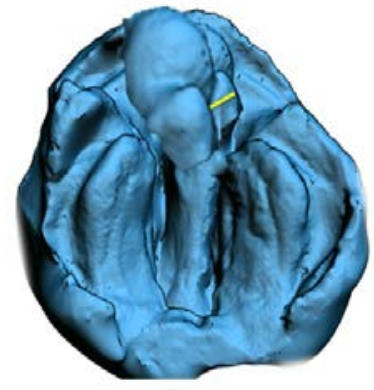

B

Figure (2): Designing phase (measuring and the angle of deviation and the showing the expected position of the premaxilla (the yellow line indicates the distance between the actual and the expected position of the premaxilla).

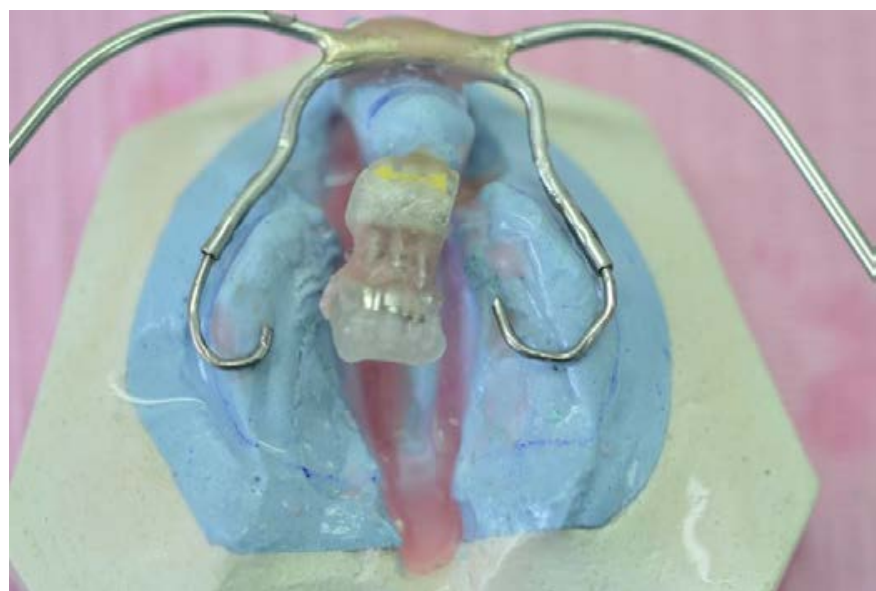

Figure (3): Adaptation of the opened expansion screw to the premaxilla.

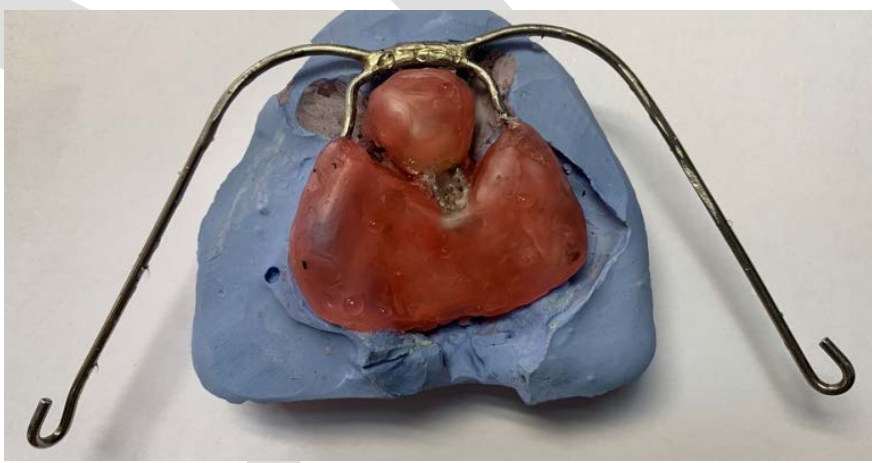

Figure (4): Wax pattern of the two piece appliance.

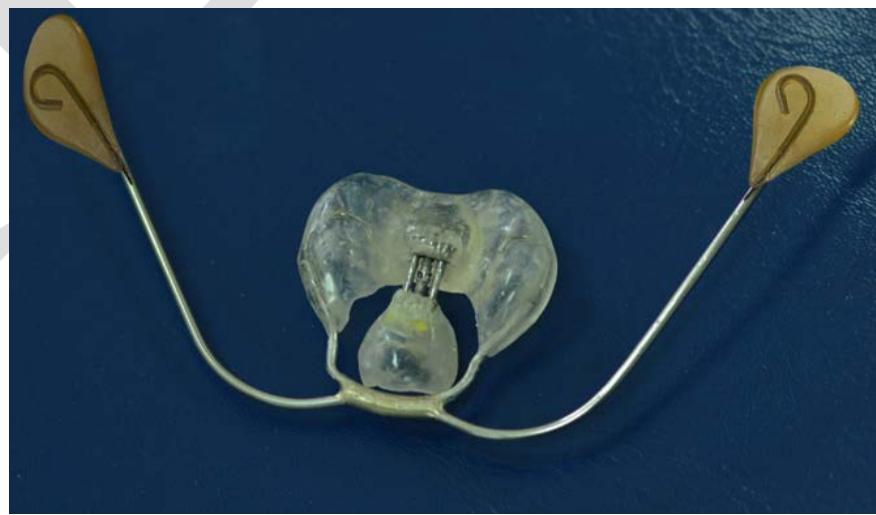

Figure (5): The final acrylic two-piece the appliance. 


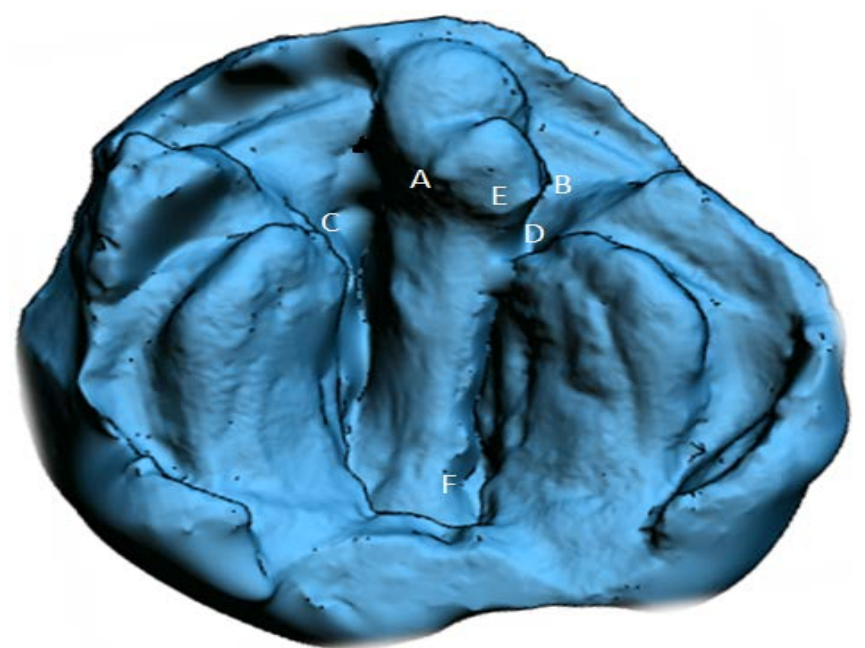

Figure (6): Landmarks of the maxillary measurements. (AC and BD) the cleft gaps (right and left) (AB) The width of the premaxilla. (EE) premaxillary protrusion.

\section{RESULTS}

Table 1 shows the change of the antroposterior retraction of the premaxilla and premaxilla width at the three time intervals. It shows statistically significant increase of the width of the premaxilla with observed increase since starting the treatment compared to 6-8 weeks of treatment and 12-16 weeks of treatment with mean value of $11.342 \pm 2.810$, $12.016 \pm 3.026$ and $12.614 \pm 3.096$ respectively with $P$ value of 0.000 . It also shows the change in the antroposterior retraction of the premaxilla with statistical significant retraction of $p$ value of $\mathrm{p}=0.000$. The mean values at the three time intervals M0, M1 and M2 were found to be 27.741a \pm 5.291 , $25.374 b \pm 5.129$ and $24.735 c \pm 5.157$. The results show that the position of the premaxilla changed with statistical significant difference.

Table 2 shows the change in the right and left cleft gap at the three interval times. It reports no statistically significant change in the width of the right and left cleft gaps with $P$ value of 0.865 and 0.855 respectively in spite of the favorably observed gap closure.

Table (1): The change in width and antroposterior retraction of the premaxilla through three time intervals.

$\mathrm{n}$ : Number of patients

Min-Max: Minimum - Maximum

CI: Confidence interval

$\mathrm{df}=$ degree of freedom

Different superscript letters indicate statistically significant difference (Adjustment for multiple comparisons using Bonferroni method).

*: Statistically significant $(\mathrm{p}<0.05)$

NS: Statistically not significant $(\mathrm{p} \geq 0.05)$

\begin{tabular}{|c|c|c|}
\hline & $\begin{array}{l}\text { Antro-posterior } \\
\text { retraction (EF) } \\
(n=15)\end{array}$ & $\begin{array}{c}\text { Premaxilla width } \\
\text { (AB) } \\
\text { (n=15) }\end{array}$ \\
\hline 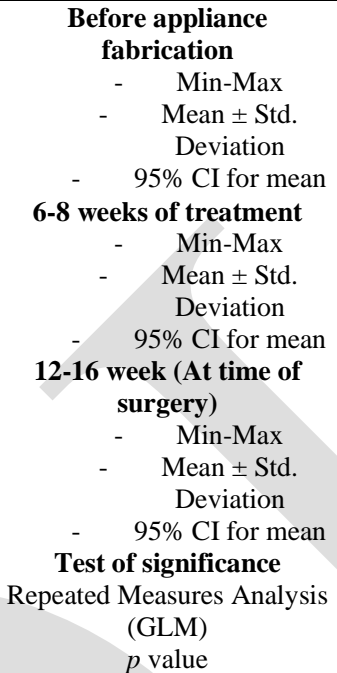 & $\begin{array}{c}18.108-38.245 \\
25.374^{\mathbf{b}} \pm 5.129 \\
24.81117-30.67083 \\
\\
16.508-37.354 \\
24.735^{\mathbf{c}} \pm 5.157 \\
22.53364-28.21476\end{array}$ & $\begin{array}{c}6.751-16.003 \\
12.016^{\mathrm{b}} \pm 3.027 \\
10.339-13.692 \\
\\
6.998-16.999 \\
12.614^{\mathrm{c}} \pm 3.096 \\
10.899-14.328\end{array}$ \\
\hline
\end{tabular}

Table (2): The change in right and left cleft gap width through three time intervals.

\begin{tabular}{|c|c|c|}
\hline & $\begin{array}{c}\text { Right cleft gap (AB) } \\
(n=15)\end{array}$ & $\begin{array}{c}\text { left cleft gap (BD) } \\
(n=15)\end{array}$ \\
\hline $\begin{array}{cc}\text { Before appliance fabrication } \\
-\quad \text { Min-Max } \\
-\quad \text { Mean } \pm \text { Std. } \\
-\quad \text { Deviation } \\
\quad \text { 95\% CI for mean }\end{array}$ & $\begin{array}{c}2.106-16.224 \\
9.421^{\mathbf{a}, \mathbf{b}, \mathbf{c}} \pm 4.467 \\
6.94793-11.89501\end{array}$ & $\begin{array}{c}2.146-19.642 \\
9.650^{\mathbf{a}, \mathbf{b}, \mathbf{c} \pm 5.606} \\
6.545-12.754\end{array}$ \\
\hline $\begin{array}{cc}\text { 6-8 weeks of treatment } \\
-\quad \mathrm{n} \\
-\quad \text { Min-Max } \\
-\quad \text { Mean } \pm \text { Std. } \\
\text { Deviation } \\
-\quad 95 \% \text { CI for mean }\end{array}$ & $\begin{array}{c}7.547-12.559 \\
9.525^{\mathbf{a}, \mathbf{b}, \mathbf{c}} \pm 1.531 \\
8.67739-10.37355\end{array}$ & 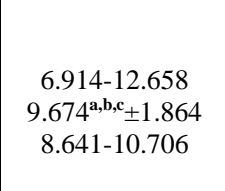 \\
\hline $\begin{array}{c}\text { 12-16 week (At time of } \\
\text { surgery) } \\
-\quad \text { Min-Max } \\
-\quad \text { Mean } \pm \text { Std. } \\
-\quad \text { Deviation } \\
\text { 95\% CI for mean } \\
\text { Test of significance } \\
\text { Repeated Measures Analysis } \\
\text { (GLM) } \\
p \text { value }\end{array}$ & 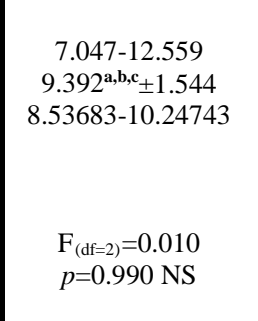 & $\begin{array}{c}6.999-12.658 \\
9.875^{\mathbf{a}, \mathbf{b}, \mathbf{c} \pm 1.897} \\
8.824-10.925\end{array}$ \\
\hline
\end{tabular}

$\mathrm{n}$ : Number of patients

Min-Max: Minimum - Maximum

CI: Confidence interval

$\mathrm{df}=$ degree of freedom

Different superscript letters indicate statistically significant difference (Adjustment for multiple comparisons using

Bonferroni method).

* : Statistically significant $(\mathrm{p}<0.05)$

NS: Statistically not significant $(\mathrm{p} \geq 0.05)$ 


\section{DISCUSSION}

Although the rehabilitation of complete bilateral cleft lip and palate CBCLP infants is complicated, the early intervention and the proper protocol of treatment make the procedure much more effective.

This study was conducted on 15 CBCLP to evaluate the efficiency of a modified two piece appliance which was used to actively mold the premaxillary tissues. The retention of the appliance was gained through extra-oral side extension arms and head-cap

Infants were chosen to be non-syndromic cases with less than 1 month old because during the period of the first 6 weeks of life there are high levels of maternal estrogen in the fetal circulation which triggers an increase in the hyaluronic acid. According to Hamrick in 1999 (26) hyaluronic acid alters the cartilage, ligament and connective tissue elasticity by breaking down intercellular matrix and this process thought to be factor for better activation of our appliance . Levels of estrogen start dropping at 6 weeks of age. The increased risk associated with syndromes suggests that the cause of excess mortality is complex so that syndromic cases were excluded (27).

Alveolar molding was selected to be used with and an active theory because according to Suri and Tompson 2004 (10), active appliances move alveolar cleft segments in a predetermined manner with controlled forces, while studies which depend on the passive theory rely it on the fact that passive appliances deliver no force but act as a fulcrum upon which the forces created by surgical lip closure contour and mold the alveolar segments in a predictable fashion.

The two piece appliance was also modified by adding side tension arms together with head cap as a means of extraoral retention according to the suggestion of Abdel Razek 1980 (16) which has good results of retention without hurting the infants' skin. Although it was showed in the literature that the most common means of retention of the NAM appliances were the extraoral tape, they reported that tape is usually associated with skin irritation and allergic reactions $(10,12-14)$ that is why side tension arms and head cap were used to increase the appliance retention and overcome the side effects of the tape.

In spite of non-significant results of reduction of the cleft gaps, they were favorably retracted and aligned to be ready for the surgical repair. These positive results can be attributed to the multidirectional forces applied by the two piece active appliance. These attributions were supported by studies which reported that the active appliances can effectively diminish the cleft gaps in both bilateral and unilateral cleft infants (13-15). The favorable cleft gap reduction was found to be statistically non-significant. This could be explained by the wide range of variability in the cleft gaps between the infants and the different direction and deviation degrees of the premaxilla in each case.

The decrease in the antro-posterior retraction of the premaxilla also supports the conclusion that the active appliance can retrude the premaxilla and close the cleft gaps.
This could be attributed to the good retention of the appliance thus the active screw was able to retrude the premaxilla to the favorable position without any skin irritation or inflammation.

\section{CONCLUSIONS}

From the previous results, we concluded that the modified pre-surgical active appliance was effective in re-alignment and retrusion the severely deviated and severely protruded premaxilla. Also it was found that side extension arms with head cap were an effective method of retaining the appliance in place.

\section{STATEMENT OF CONFLICT OF INTEREST:}

The authors declare that they have no conflicts of interest

\section{ACKNOWLEDGMENTS}

Many thanks to Dr. Mohammed Tala't El-shammy and EJUST where all the casts were scanned and all measurement had been done and evaluated.

\section{REFERENCES}

1. Murray J. Gene/ Environment causes of cleft lip and/or palate. Clin Genet. 2002;61:248-56.

2. Bender P. Genetics of cleft lip and palate. $\mathrm{J}$ Pediatr Nurs. 2000;15:242-9.

3. Chai Y, Maxson RE Jr. Recent advances in craniofacial morphogenesis. Dev Dyn. 2006;235:2353-75.

4. Amaratunga NA. A study of etiologic factors for cleft lip and plate in Sri Lanka. J Oral Maxillofac Surg. 1989;47:710.

5. Butali A, Little J, Chevrier C, Cordier S, SteegersTheunissen R, Jugessur A, et al. Folic acid supplementation use and the MTHFR C677T polymorphism in orofacial clefts etiology: An individual participant data pooledanalysis. Birth Defects Res A Clin Mol Teratol. 2013;97:509-14.

6. Chung MK, Lao TT, Ting YH, Leung TY, Lau TK, Wong TW. Environmental factors in the first trimester and risk of oral-facial clefts in the offspring. Reprod Sci. 2013;20:797803.

7. CH Thorne, RW Beasley, SJ Aston, SP Bartlett, GC Geoffrey, SL Scott. Grabb and Smith's Plastic Surgery, $6^{\text {th }}$ ed. USA: Lippincott Williams \& Wilkins; 2007.

8. Aminpour S, Tollefson TT. Recent advances in presurgical molding in cleft lip and palate. Curr Opin Otolaryngol Head Neck Surg. 2008;16:339-46.

9. Grayson BH, Garfinkle JS. Early cleft management: The case for nasoalveolar molding. Am J Orthod Dentofacial Orthop. 2014;145:134-42.

10. Suri S, Tompson BD. A modified muscle-activated maxillary orthopedic appliance for presurgical nasoalveolar molding in infants with unilateral cleft lip and palate. Cleft Palate Craniofac J. 2004;41:225-9.

11. Neligan PC, Buck DW. Core Procedures in Plastic Surgery. Philadelphia : Saunders; 2013. 
12. Retnakumari N, Santhakumar M. A new approach in presurgical infant orthopedics using an active alveolar moulding appliance in the management of bilateral cleft lip and palate patient: A case report. IOSR J Dent Med Sci. 2013;12:11-5.

13. García LG, Rodríguez EG, Yudovich Burak M, Saavedra MDLPA, García-López S, Arriaga REV. Pre-orthopedic appliance with pins used in alignment of maxillary segments in patients with unilateral cleft lip and palate. Revista Odontológica Mexicana. 2014;18:222-8.

14. Kiya K, Oyama T, Sone Y, Ishii N, Hosokawa K. A novel active intraoral appliance for presurgical orthopaedic treatment in patients with complete bilateral cleft lip and palate. J Plast Reconstr Aesthet Surg 2015;68:632-7.

15. Grayson B H, Santiago P E. Pre Surgical Orthopaedics for Cleft Lip and Palate. Grabb and Smith's Plastic Surgery. 5th ed. Philadelphia: Lippin Cott Raven Publishers; 1997. p237-44.

16. Razek MK. Prosthetic feeding aids for infants with cleft lip and palate. J Prosthet Dent 1980;44:556-61.

17. Hertzog MA. Considerations in determining sample size for pilot studies. Res Nurs Health. 2008;31:180-91.

18. Oosterkamp BC, van Oort RP, Dijkstra PU, Stellingsma K, Bierman MW, de Bont LG. Effect of an intraoral retrusion plate on maxillary arch dimensions in complete bilateral cleft lip and palate patients. Cleft Palate Craniofac J. 2005;42:239-44.
19. Agrawal R, Patel D. Orthopaedic traction with passive nasoalveolar moulding in a bilateral cleft lip and palate patient - Rediscovering the old. JPRAS. 2019;19:50-5.

20. IBM Corp. IBM SPSS Statistics for Windows, Version 21.0. Armonk, NY: IBM Corp.; Released 2012.

21. Field A. Discovering Statistics Using IBM SPSS Statistics. 4th ed. London, California, New Delhi: SAGE Publications Ltd; 2013.

22. Snecdecor GW, Cochran WG. Statistical Methods: Wiley; 1991.

23. Box JF. Guinness, Gosset, Fisher, and small samples. Statistical science. 1987: 45-52.

24. Welch BL. The generalization ofstudent's' problem when several different population variances are involved. Biometrika. 1947; 34: 28-35.

25. Ruxton GD. The unequal variance t-test is an underused alternative to Student's t-test and the Mann-Whitney U test. Behav Ecol. 2006; 17: 688-90.

26. Hamrick MWJ. A chondral modeling theory revisited. Theor Biol. 1999; 201:201-8.

27. Christensen K, Juel K, Herskind AM, Murray JC. Long term follow up study of survival associated with cleft lip and palate at birth. BMJ. 2004;328:53-9. 